\title{
Single Particle Motions in Liquids: Qualitative Features of Memory Functions
}

\author{
Raymond D. Mountain \\ Institute for Basic Standards, National Bureau of Standards, Washington, D.C. 20234
}

(February 1, 1974)

\begin{abstract}
Memory functions, which enter into the equations of motion for time correlation functions, are constructed from neutron scattering, infrared absorption and light scattering data involving single particle motions in liquids. The qualitative features of these memory functions are related to the shape of the corresponding time correlation functions. It is found that a negative portion to the memory function is indicative of a rapid loss of correlation in time while strong temporal correlations imply a memory function which does not go negative. The mathematical structure of a memory function is examined for the case of the ideal gas by expanding and evaluating the projection operator representation of the function. The resulting expression has a rich mathematical structure and can be expressed in a closed form only for its Laplace transform.
\end{abstract}

Key words: Depolarized Rayleigh scattering; ideal gas; incoherent neutron scattering; infrared absorption; liquid state; memory function; projection operator; Raman scattering; time correlation function.

\section{Introduction}

The time correlation of molecular quantities has proven to be a useful way of describing molecular motions in liquids. These time correlation functions, which are obtained as Fourier transforms of data, can be investigated using recently developed techniques of time dependent statistical mechanics [1]. Exact equations of motion for the time correlation functions have been derived which involve memory functions, quantities which characterize the history of the fluid $[1,2]$. While time correlation functions are now widely used to represent molecular motions [3], only a few studies of memory functions have appeared [4-9]. In this paper we examine some properties of memory functions as derived from data and from model correlation functions. Our results, combined with other results obtained from computer simulations, enable us to make some qualitative observations about the general features of these important quantities.

A motivation for studying memory functions is the hope that these quantities will have simpler structure than the time correlation functions. Should this be true, it would be possible to model molecular motions in liquids in terms of a relatively simple memory function once the qualitative features of the memory functions are understood. Indeed, this has been done empirically for the velocity time correlation functions obtained by computer simulation of the LennardJones model fluid [10].

The mathematical structure of memory functions has been studied in the short time and long time limits [4-6]. We present here a calculation for all times of the memory function for the incoherent neutron scattering by an ideal gas. The mathematical structure of this quantity is found to involve even powers of the velocity of a particle in terms of deviations of the even powers from their average values. This structure is more complex than the structure of the time correlation function. Thus we have examples which suggest the existence of a rich underlying mathematical structure which yields relatively simple functional forms for the memory functions.

It is plausible to expect that future studies will find a whole range of cases and that for as yet to be determined physical situations, the memory functions are comparatively simple quantities.

The paper is organized in the following way. First we introduce some notation and outline briefly the relation connecting a time correlation function and a memory function. In section 3 we construct memory functions from data and discuss the structural features of these quantities. The mathematical structure of memory functions is probed in section 4 where the memory function for the incoherent scattering from an ideal gas is calculated from the microscopic definition of a memory function. The structure discovered in this analysis is found to have some unexpected features. The discussion of the qualitative features of memory functions in section 5 makes use of both our results and the results of other studies.

\section{Formal Preliminaries}

Molecular motions in liquids may be studied experimentally in a number of ways. For example, the 
translational motion of a single atom is probed by incoherent neutron scattering and the changes in orientation of a single molecule is probed by depolarized Rayleigh scattering. Infrared absorption and certain types of depolarized Raman scattering also probe orientational motions. In each case one can obtain from the data, a time correlation function which describes some aspect of the motion of a typical molecule in the fluid.

The notation we shall employ for a time correlation function is $\psi(t)$ with $\psi(0)=1$ where

$$
\psi(t)=\left\langle u^{*}(0) u(t)\right\rangle /\left\langle|u|^{2}\right\rangle .
$$

Here the angular brackets $\langle\ldots\rangle$ indicate an ensemble average and the phase function

$$
u(t)=\exp (-i L t) u(0)
$$

evolves in time as determined by the Liouville operator, $L$, for the system. The phase functions associated with various experimental probes are described elsewhere [11].

A formally exact equation of motion for $\psi(t)$ may be derived using the projection operator formalism of Zwanzig $[1,2]$. The equation is

$$
\frac{\partial \psi(t)}{\partial t}=-\int_{0}^{t} d t^{\prime} K\left(t-t^{\prime}\right) \psi\left(t^{\prime}\right)
$$

with

$$
\left.\frac{\partial \psi}{\partial t}\right|_{t=0}=0
$$

The memory function $K(t)$ is specified as

$$
K(t)=\left\langle\dot{u}^{*}(0) \exp [-(1-\mathrm{P}) i L t] \dot{u}(0)\right\rangle
$$

where the projection operator $P$ has the property

$$
P g=u\left\langle u^{*} g\right\rangle
$$

for any phase function $g$ and

$$
\dot{u}=-i L u \text {. }
$$

\section{Memory Functions}

In this paper we examine memory functions obtained by recursively solving eq (2.3) for $K(t)$ when $\psi(t)$ is known from experiment. The numerical procedures are discussed in the appendix. We use incoherent neutron scattering data for liquid argon [12] as an example of translational motion. Infrared absorption data and depolarized Raman scattering data for neopentane [13] and depolarized Rayleigh scattering data for benzene [14] are used as examples of orientational motion.

The results are displayed in figures $1-4$. There the correlation function, $\psi(t)$, and the normalized memory

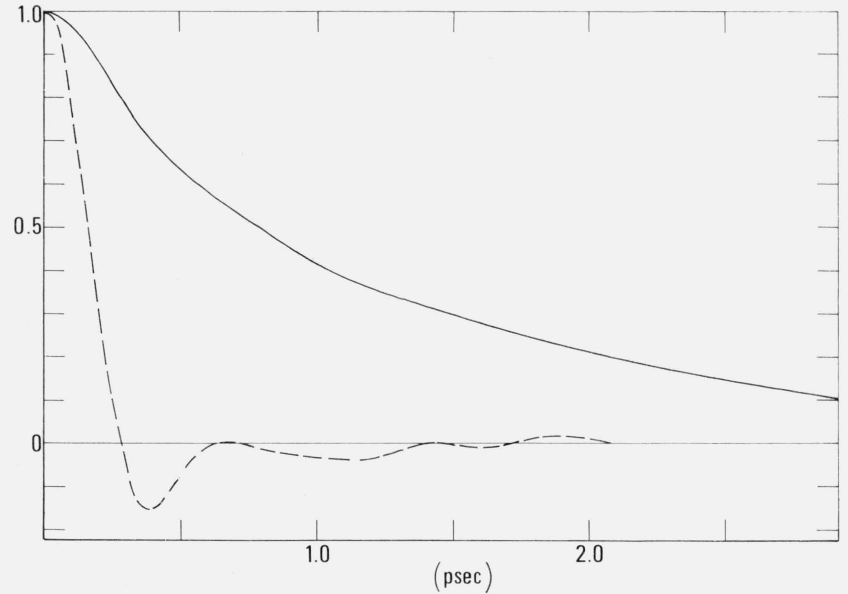

FIGURE 1. Single particle translation motion for liquid argon at $\mathrm{T}=85.2 \mathrm{~K}$.

The time correlation function $(-)$ and normalized memory function $(---)$ for $Q$ $=2.0 \AA^{-1}$ are pictured.

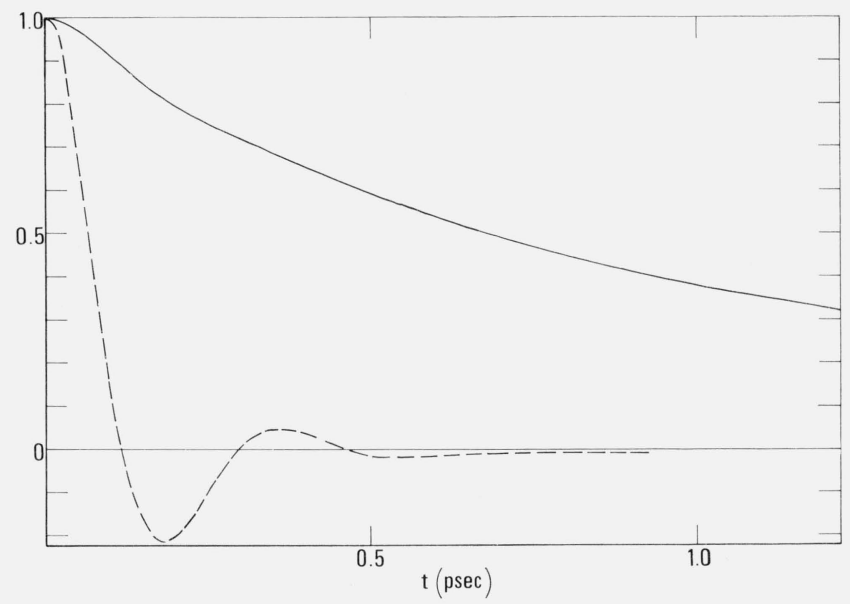

FiguRE 2. Reorientation of liquid benzene molecules at $\mathrm{t}=59{ }^{\circ} \mathrm{C}$ studied via depolarized Rayleigh scattering.

The $l=2$ component of reorientation motion is pictured. The time correlation function $(-)$ and the normalized memory function (-- ) are shown as functions of time.

function, $K(t) / K(0)$, are shown as functions of time. These memory functions are characterized by a rapid initial decrease to negative values followed by an approach to zero which is prompt when compared with the decay of the correlation function. This appears to be a fairly common situation as evidenced by its presence in the memory function for the incoherent neutron scattering from an ideal gas as shown in figure 5. It is not a universal feature as counter examples may be constructed.

The results for $K(t)$ become erratic for long times. This is a result of the sensitivity to irregularities in $\psi(t)$ of the numerical procedure used to obtain $K(t)$. The uncertainty of $K(t)$ in figure 1 is on the order of \pm 0.02 for times greater than $10^{-12} \mathrm{~s}$. The pair of dashed lines in figure 3 indicate the range of values assumed by $K(t)$. In figure 4 the solid horizontal lines 
indicate the range of values obtained for $K(t)$ for times greater than $0.8 \times 10^{-12} \mathrm{~s}$. The data used to obtain figure 2 were carefully smoothed so the erratic behavior does not show up until longer times are reached and would not be visible with this scale.

Figure 5 shows the memory function associated with a Gaussian time correlation function (ideal gas). Here the negative region is not as pronounced as in liquid argon but it is definitely there.

A model time correlation function which does not have a negative portion to its memory function is

$$
\psi(t)=\exp \left[A-\sqrt{A^{2}+t^{2} / \tau^{2}}\right] .
$$

The results for $A=1.0$ and $\tau=0.5$ are displayed in figure 6 . The tail of the memory function is approximately exponential.

The existence of a negative portion in the memory function is not surprising. From eq (2.5) we observe that $C(t)$, the negative of the second time derivative of $\psi(t)$, is not dissimilar to $K(t)$ since

$$
\begin{aligned}
C(t) & =-\partial^{2} \psi(t) / \partial t^{2} \\
& =\left\langle\dot{u}^{*} \exp [-i L t] u\right\rangle .
\end{aligned}
$$

At least for short times, $K(t)$ and $C(t)$ should be fairly close in value. They are identical at $t=0$. The function $C(t)$ necessarily has negative portions since

$$
\int_{0}^{\infty} d t C(t)=0 \text {. }
$$

This follows readily from the initial condition $\partial \psi(t) /$ $\left.\partial t\right|_{t=0}=0$. By taking Laplace transforms we obtain

$$
\begin{aligned}
\hat{C}(z) & =\int_{0}^{\infty} d t e^{-z t} C(t) \\
& =-z^{2} \hat{\psi}(z)+z
\end{aligned}
$$

so that

$$
\int_{0}^{\infty} d t C(t)=\lim _{z \rightarrow 0} \hat{C}(z)=0 .
$$

Let us now examine further the relationships between $C(t)$ and $K(t)$. The operator identity

$$
\begin{gathered}
\exp [-(1-P) i L t]=\exp [-i L t] \\
+\int_{0}^{t} d t^{\prime} \exp \left[-i L\left(t-t^{\prime}\right)\right] i P L \exp \left[-i(1-P) L t^{\prime}\right]
\end{gathered}
$$

may be used to show that

$$
K(t)=C(t)-\int_{0}^{t} d t^{\prime} K\left(t-t^{\prime}\right) \partial \psi\left(t^{\prime}\right) / \partial t^{\prime} .
$$

This equation may also be obtained directly from eq (2.3). In some of the cases considered in this article, the second term in eq (3.7) is numerically small compared to $K(t)$ for short times. This suggests that $K(t) \approx C(t)$ might be a useful starting point for an iterative solution of eq (2.3). Unfortunately this is not the case as only the trivial solution $\psi(t)=1$ exists when $K(t)$ is replaced by $C(t)$.

Another limitation of this way of examining $K(t)$ only becomes apparent after the mathematical structure of $K(t)$ is studied by evaluating eq (2.5) for incoherent neutron scattering by an ideal gas. This is done in the next section. The point to be made here is that the decomposition of the memory function implied by eq (3.7) is not particularly useful for evaluating $K(t)$, at least for this example.

\section{Ideal Gas Memory Function}

In this section we examine the memory function for incoherent neutron scattering by an ideal gas. The phase function $u$ is $\exp (i \mathbf{q} \cdot \mathbf{r})$ where $\mathbf{q}$ is the momentum transfer and $\mathbf{r}$ is the position of a gas atom. It is a simple matter to show that for the ideal gas

$$
\begin{gathered}
\psi(t)=\left\langle e^{-i \mathbf{q} \cdot \mathbf{r}} e^{-i L t} e^{i \mathbf{q} \cdot \mathbf{r}}\right\rangle \\
=\langle\cos (\mathbf{q} \cdot \mathbf{v} t)\rangle=\exp \left[-q^{2} t^{2} / 2 \beta m\right]
\end{gathered}
$$

where $m$ is the mass of the gas atom and $\beta=1 / k_{B} T$, the inverse of Boltzmann's constant $k_{B}$ times the absolute temperature $T$.

The memory function is formally

$$
K(t)=\left\langle e^{-i \mathbf{q} \cdot \mathbf{r}}(-i \mathbf{q} \cdot v) e^{-(1-P) i L t}(i \mathbf{q} \cdot \mathbf{v}) e^{i \mathbf{q} \cdot \mathbf{r}}\right\rangle .
$$

The evaluation of this expression is now to be undertaken. The procedure we follow is to expand the exponential factor $\exp [-i(1-P) L t]$, apply the operator $-i(1-P) L$ the appropriate number of times and then collect the resulting terms in such a way that a closed form is obtained for the Laplace transform of $K(t)$. It does not seem to be possible to obtain a closed form for $K(t)$ itself.

For the ideal gas, the dynamics are trivial;

$$
\begin{aligned}
& -i L \mathbf{r}=\mathbf{v} \\
& -i L \mathbf{v}=0 .
\end{aligned}
$$

Let us consider first the expansion of $K(t)$ :

$$
\begin{gathered}
K(t)=\left\langle e^{-i \mathbf{q} \cdot \mathbf{r}}(-i \mathbf{q} \cdot \mathbf{v}) \sum_{n=0}^{\infty}\right. \\
(-t)^{n}[(1-P) i L]^{n} \\
n !
\end{gathered}
$$

We note that the odd terms in $t$ will vanish when the expectation value is taken. First consider the $n=2$ coefficient.

$$
[(1-P) i L]^{2}(i \mathbf{q} \cdot \mathbf{v}) e^{i \mathbf{q} \cdot \mathbf{r}}
$$




$$
\begin{aligned}
& =(1-P) i L\left[(\mathbf{q} \cdot \mathbf{v})^{2}-\left\langle(\mathbf{q} \cdot \mathbf{v})^{2}\right\rangle\right] e^{i \mathbf{q} \cdot \mathbf{r}} \\
& =\left[(\mathbf{q} \cdot \mathbf{v})^{2}-\left\langle(\mathbf{q} \cdot \mathbf{v})^{2}\right\rangle\right](-i \mathbf{q} \cdot \mathbf{v}) e^{i \mathbf{q} \cdot \mathbf{r}} \\
& =k_{2}(-i \mathbf{q} \cdot \mathbf{v}) e^{i \mathbf{q} \cdot \mathbf{r}} .
\end{aligned}
$$

Repeated application of $[(1-P) i L]^{2}$ yields a sequence of terms of the type

$$
(-1)^{n} k_{2 n}(i \mathbf{q} \cdot \mathbf{v}) e^{i \mathbf{q} \cdot \mathbf{r}}
$$

where the coefficients $k_{2 n}$ satisfy

$$
k_{2 n+2}=k_{2 n}(\mathbf{q} \cdot \mathbf{v})^{2}-\left\langle k_{2 n}(\mathbf{q} \cdot \mathbf{v})^{2}\right\rangle
$$

and

$$
k_{0}=1 \text {. }
$$

A more useful grouping of terms is achieved by introducing another set of coefficients $\left\{C_{j}\right\}$ defined by

$$
k_{2 n}=\sum_{j=0}^{n-1} C_{j}\left(x^{2 n-2 j}-\left\langle x^{2 n-2 j}\right\rangle\right)
$$

with $C_{0}=1$. The notation $x=\mathbf{q} \cdot \mathbf{v}$ has been introduced to simplify the writing. The $C_{j}$ 's satisfy the recursion relation

$$
C_{n}=-\sum_{j=0}^{n-1} C_{j}\left\langle x^{2 n-2 j}\right\rangle .
$$

Now let us express $K(t)$ in terms of the $C_{j}$ 's. The factors $\exp (i \mathbf{q} \cdot \mathbf{r})$ yield 1 when the expectation value is taken so we drop those terms.

$$
\begin{aligned}
K(t) & =\left\langle x^{2} \sum_{n=0}^{\infty} \frac{\left(-t^{2}\right)^{n}}{(2 n) !} k_{2 n}\right\rangle \\
& =\left\langle x^{2}\right\rangle+\left\langle x^{2} \sum_{n=1}^{\infty} \frac{\left(-t^{2}\right)^{n}}{(2 n) !} \sum_{j=0}^{n-1} C_{j}\left(x^{2 n-2 j}-\left\langle x^{2 n-2 j}\right\rangle\right)\right\rangle
\end{aligned}
$$

$$
=\left\langle x^{2}\right\rangle+\left\langle\left(x^{2}-\left\langle x^{2}\right\rangle\right) \sum_{n=1}^{\infty} \sum_{j=0}^{n-1} \frac{\left(-t^{2}\right)^{n}}{(2 n) !} C_{j} x^{2 n-2 j}\right\rangle .
$$

It will prove convenient to reorder the double sum in eq (4.9) so that

$$
\begin{aligned}
K(t)= & \left\langle x^{2}\right\rangle+\left\langle\left(x^{2}-\left\langle x^{2}\right\rangle\right) \sum_{j=0}^{\infty} \sum_{n=j+1}^{\infty} C_{j} \frac{\left(-t^{2}\right)^{n}}{(2 n) !} x^{2 n-2 j}\right\rangle \\
= & \left\langle x^{2}\right\rangle+C_{0}\left\langle\left(x^{2}-\left\langle x^{2}\right\rangle\right) \sum_{n=1}^{\infty} \frac{\left(-t^{2}\right)^{n}}{(2 n) !} x^{2 n}\right\rangle \\
& +\left\langle\left(x^{2}-\left\langle x^{2}\right\rangle\right) \sum_{j=1}^{\infty} \sum_{n=j+1}^{\infty} C_{j} \frac{\left(-t^{2}\right)^{n}}{(2 n) !} x^{2 n-2 j}\right\rangle .
\end{aligned}
$$

The next step in the analysis involves the use of eq (4.8) to systematically replace the $C_{j}$ 's in eq (4.10) with $C_{0}$.
Thus we find

$$
\begin{aligned}
& K(t)=\left\langle x^{2}\right\rangle+C_{0} g_{1}(t) \\
& -\left\langle\left(x^{2}-\left\langle x^{2}\right\rangle\right) \sum_{j=1}^{\infty} \sum_{n=j+1}^{\infty} \sum_{k=0}^{j-1} C_{k}\left\langle x^{2 j-2 k}\right\rangle \frac{\left(-t^{2}\right)^{n}}{(2 n) !} x^{2 n-2 j}\right\rangle .
\end{aligned}
$$

Now reorder the $j$ and $k$ sums and extract the $k=0$ term to obtain

$$
\begin{aligned}
& K(t)=\left\langle x^{2}\right\rangle+C_{0} g_{1}(t)-C_{0} g_{2}(t) \\
& -\left\langle\left(x^{2}-\left\langle x^{2}\right\rangle\right) \sum_{k=1}^{\infty} \sum_{j=k+1}^{\infty} \sum_{n=j+1}^{\infty} C_{k}\left\langle x^{2 j-2 k}\right\rangle \frac{\left(-t^{2}\right)^{n}}{(2 n) !} x^{2 n-2 j}\right\rangle .
\end{aligned}
$$

This process can be repeated with the result

$$
K(t)=\left\langle x^{2}\right\rangle-C_{0} \sum_{N=1}^{\infty}(-1)^{N} g_{N}(t)
$$

where

$$
\begin{aligned}
& g_{N}(t)=\left\langle\left(x^{2}-\left\langle x^{2}\right\rangle\right) \sum_{k_{1}=1}^{\infty} \sum_{k_{2}=k_{1}+1}^{\infty}\right. \\
& \ldots \sum_{n=k_{N-1}+1}^{\infty} \frac{\left(-t^{2}\right)^{n}}{(2 n) !}\left\langle x^{2 k_{1}}\right\rangle\left\langle x^{2 k_{2}-2 k_{1}}\right\rangle \\
& \text {.. }\left\langle x^{2 k_{N-1}-2 k_{N-2}}\right\rangle\left\langle x^{2 n-2 k_{N-1}}\right\rangle \text {. }
\end{aligned}
$$

Further progress is possible if we make use of the moment property of the $x$ 's for the ideal gas, namely

$$
\left\langle x^{2 j}\right\rangle=(2 j-1) ! !\left\langle x^{2}\right\rangle^{j} .
$$

The $(2 n)$ ! term in the denominator can be removed by taking Laplace transforms [15]. For the case $N=1$, we obtain

$$
\hat{g}_{1}(z)=\int_{0}^{\infty} d t e^{-z t} g_{1}(t)
$$

$$
\begin{aligned}
\left.=\left\langle x^{2}-\left\langle x^{2}\right\rangle\right) \quad \sum_{n=1}^{\infty} \frac{(-1)^{n} x^{2 n}}{z^{2 n+1}}\right\rangle & \\
& =\left\langle\frac{x^{4}-x^{2}\left\langle x^{2}\right\rangle}{z^{3}} \frac{1}{1+x^{2} / z^{2}}\right\rangle .
\end{aligned}
$$

Closed forms for the other $\hat{g}_{j}(z)$ are also possible. We evaluate $\hat{g}_{2}(z)$ to illustrate how this is done. First we use eqs $(4.14,4.15)$ to obtain

$\hat{g}_{2}(z)=\left\langle\left(x^{2}-\left\langle x^{2}\right\rangle\right) \sum_{j=1}^{\infty} \sum_{n=j+1}^{\infty}(2 j-1) ! !\right.$

$$
\left.\frac{\left\langle x^{2}\right\rangle^{j}(-1)^{n} x^{2 n-2 j}}{z^{2 n+1}}\right\rangle
$$




$$
\begin{aligned}
& =\left\langle\frac{\left(x^{2}-\left\langle x^{2}\right\rangle\right)}{z} \sum_{j=1}^{\infty}(2 j-1) ! !\left[\frac{\left\langle x^{2}\right\rangle}{x^{2}}\right]^{j} \frac{\left(-x^{2} / z^{2}\right)^{j+1}}{1+x^{2} / z^{2}}\right\rangle \\
& =\left\langle\frac{x^{4}-x^{2}\left\langle x^{2}\right\rangle}{z^{3}\left(1+x^{2} / z^{2}\right)}\right\rangle \sum_{j=1}^{\infty}(2 j-1) ! !(-1)^{j+1}\left[\left\langle x^{2}\right\rangle / z^{2}\right]^{j}
\end{aligned}
$$

Next we make use of the result

$$
(2 j-1) ! !=\sqrt{\frac{2}{\pi}} \int_{0}^{\infty} d q q^{2 j} \exp \left(-q^{2} / 2\right)
$$

to obtain

$$
\hat{g}_{2}(z)=\left\langle\frac{x^{4}-x^{2}\left\langle x^{2}\right\rangle}{z\left(z^{2}+x^{2}\right)}\right\rangle \hat{I}(z)
$$

where

$$
\hat{I}(z)=\sqrt{\frac{2}{\pi}} \int_{0}^{\infty} d q \frac{e^{-q^{2} / 2}\left\langle x^{2}\right\rangle q^{2}}{z^{2}+\left\langle x^{2}\right\rangle q^{2}}
$$

It is a straightforward task using these techniques to show that

$$
\hat{g}_{N}(z)=(-1)^{N+1} \hat{g}_{1}(z)[\hat{I}(z)]^{N-1} .
$$

Next, the Laplace transform of the memory function is found to be

$$
\hat{K}(z)=\frac{\left\langle x^{2}\right\rangle}{z}+\frac{\hat{g}_{1}(z)}{1-\hat{I}(z)} .
$$

This in turn is shown to be

$$
\begin{aligned}
\hat{K}(z) & =-z+\left[\sqrt{\frac{2}{\pi\left\langle x^{2}\right\rangle}}\right. \\
& \left.\times \exp \left(z^{2} / 2\left\langle x^{2}\right\rangle\right) \operatorname{erfc}\left(z / \sqrt{2\left\langle x^{2}\right\rangle}\right)\right]^{-1} .
\end{aligned}
$$

One may also express $\hat{K}(z)$ in terms of $\hat{\psi}(z)$ as

$$
\hat{K}(z)=-z+1 / \hat{\psi}(z)
$$

by taking Laplace transforms of eq (2.3) and solving for $\hat{K}(z)$. In terms of $\left\langle x^{2}\right\rangle$, it follows from eq (4.1) that

$$
\psi(t)=\exp \left(-\left\langle x^{2}\right\rangle t^{2} / 2\right)
$$

since

$$
\left\langle x^{2}\right\rangle=\frac{\int_{0}^{\infty} d v(q v)^{2} e^{-\beta m v^{2} / 2}}{\int_{0}^{\infty} d v e^{-\beta m v^{2} / 2}}=q^{2} / \beta m
$$

and thus

$$
\hat{\psi}(z)=\sqrt{\frac{\pi}{2\left\langle x^{2}\right\rangle}} \exp \left(z^{2} / 2\left\langle x^{2}\right\rangle\right) \operatorname{erfc}\left(z / \sqrt{2\left\langle x^{2}\right\rangle}\right)
$$

in agreement with the result of summing $\hat{K}(z)$.

Although a closed form for $K(t)$ has not been found, we have determined the underlying structure of the memory function. How it compares with the structure implied by eq (3.7) is worth noting. From eqs (4.21) and (4.22) it is evident that

$$
\hat{K}(z)=\frac{\left\langle x^{2}\right\rangle}{z}+g_{1}(z) \sum_{n=0}^{\infty}[-\hat{I}(z)]^{n} .
$$

A simple calculation shows that

and

$$
\begin{aligned}
g_{1}(t) & =C(t)-\left\langle x^{2}\right\rangle \psi(t) \\
I(t) & =\partial \psi(t) / \partial t .
\end{aligned}
$$

An iterative solution of eq (3.7) yields

$$
\hat{K}(z)=\hat{C}(z) \sum_{n=0}^{\infty}[-\hat{I}(z)]^{n} .
$$

Consistency requires that

$$
\hat{\psi}(z) \sum_{n=0}^{\infty}[-\hat{I}(z)]^{n}=1 / z .
$$

Since $\hat{I}(z)=z \hat{\psi}(z)-1$, eq (4.32) is satisfied and the two solutions are equivalent. The mathematical structure which was found to be useful for summing the memory function expansion may appear in retrospect to be inconvenient because of the extensive cancellations which necessarily occur. However, attempts to impose the structure of eq (3.7) on the expansion were unsuccessful in achieving a form which could be completely summed.

In order to see just what sort of structure we have developed, let us review the steps followed in summing the expansion of $K(t)$. The set of coefficients $\left\{k_{2 n}\right\}$ resulted from grouping the sum in terms of powers of the time. Next. the sum was arranged in terms of the deviations of $x^{2 l}$ from the average $\left\langle x^{2 l}\right\rangle$ and resulted in the set of coefficients $\left\{C_{j}\right\}$. This was the crucial step in the analysis as it exhibited the underlying structure of the memory function. Clearly this way of arranging the sum is different from the structure implied by eq (3.7) since $C(t)$ does not involve the projection operator. Next we eliminated all $C_{j}$ 's except $C_{0}$ from the expansion through repeated use of eq (4.8). The closed form obtained in eq (4.23) required an explicit relation among the moments of $x$, namely eq (4.15) which follows from the Boltzmann distribution for the velocities. It would be interesting to see if this type of structure could usefully be imposed on $K(t)$ for an interacting system. This topic is not considered here.

\section{Discussion}

The time correlation functions, $\psi(t)$, shown in figures 1-4 are typical of the ones obtained from 
liquid state scattering and absorption data. The curves initially exhibit a rapid falloff and then go over to an approximately exponential function for long times. The functions, $\psi(t)$, are everywhere positive and, except for the short time part, the curves are concave upward. The memory functions $K(t)$ exhibit a rapid initial falloff which carries them to negative values. The negative portion of $K(t)$ has the effect of decreasing the magnitude of $\partial \psi / \partial t$ and thereby reducing the slope of $\psi(t)$ so that $\psi(t)$ remains positive for all times.

It is interesting to compare this situation with that found from computer simulations for the velocity time correlation functions in Lennard-Jones liquids $[9,10]$. There the time correlation functions achieve negative values. This implies that the initial state is "strongly remembered" by the system, a viewpoint consistent with the behavior of $K(t)$ since $K(t)$ remains positive. At higher temperatures, where the kinetic energy is large compared with the strength of the pairwise interaction energy, the time correlation functions do not go negative while the memory functions do.

This suggests that the persistence of the memory function is closely related to the time evolution of the cage-like many body structure described by Rahman [16]. He found from his molecular dynamics calculations that a liquid atom undergoes a strony, velocity reversing collision and then "rattles" about in a loose cage of neighboring atoms until it escapes when the cage breaks down. The rattling motion leads to negative values of the velocity time correlation function. However, if the cage breaks up too rapidly, as it would at high temperatures or low densities, no rattling motion, coherent with the initial condition, occurs and $\psi(t)$ remains positive.

The calculation of the memory function for the ideal gas shows that this function has an extensive mathematical structure. This is expressed in the properties of the coefficients $\left\{k_{2 n}\right\}$ and $\left\{C_{j}\right\}$. The memory functions constructed from data show more structure than the memory function determined numerically from the ideal gas time correlation function.

This comparisun is the basis for the following conjecture. The mathematical structure of the memory function for an interacting system should exhibit two parts, in contrast with the one part exhibited by the ideal gas. There should be a "short-time" part which dominates the initial behavior of $K(t)$ and then goes rapidly to zero. The second part of the memory function should dominate the remainder of the time interval. This part can be either positive or negative depending on the type of correlation involved and is dominated by many-body effects which are only poorly understood. The testing of this conjecture is a subject for further work.

\section{Appendix}

The numerical inversion of eq (2.3) to obtain $K(t)$ from $\psi(t)$ is accomplished by replacing the integro- differential equation by a set of coupled difference equations defined on a discrete set of evenly spaced time points $t_{i}$ with $t_{i+1}-t_{i}=\Delta t$. For simplicity, a subscript notation is used so $\psi\left(t_{i}\right)$ becomes $\psi_{i}$ and $\psi(0)=\psi_{0}$. The trapezoidal rule and simple difference expressions are used to replace integral and differential operations. A typical member of this set of difference equations is

$$
\frac{1}{2 \Delta t}\left[\psi_{i+1}-\psi_{i-1}\right]=-\Delta t\left[\frac{1}{2}\left(K_{0} \psi_{i}+K_{i} \psi_{0}\right)+\sum_{j=1}^{i-1} K_{i-j} \psi_{j}\right]
$$

These equations for $K_{i}$ are solved recursively in terms of the $\left\{\psi_{j}\right\}$ subject to the initial conditions

$$
\begin{aligned}
& \psi_{0}=1, \psi_{1}=\psi_{-1} \\
& K_{0}=2\left(\psi_{1}-\psi_{0}\right) /(\Delta t)^{2} .
\end{aligned}
$$

The last condition follows from eqs (2.5 and 3.2) which indicate that

$$
K(0)=-\partial^{2} \psi(t) /\left.\partial t^{2}\right|_{t=0}
$$

The solution has the form

$$
\begin{aligned}
& K_{1}=-K_{0} \psi_{1}-\left[\psi_{2}-\psi_{0}\right] /(\Delta t)^{2} \\
& K_{i}=-K_{0} \psi_{i}-2 \sum_{j=1}^{i-1} K_{i-j} \psi_{j} \\
& i>1 \\
& -\left[\psi_{i+1}-\psi_{i-1}\right] /(\Delta t)^{2} .
\end{aligned}
$$

These expressions were used to obtain the $K(t)$ curves discussed in the text.

It is necessary that $\psi(t)$ be a smooth function of $t$ if this technique is to yield useful results for $K(t)$. Figures 1-4 indicate the kinds of uncertainties which show up in $K(t)$. Some care is needed in the selection of the time increment $\Delta t$. If it is too large the difference expression for the first derivative is inaccurate. If $\Delta t$ is too small, the noise in $\psi(t)$ governs the outcome rather than $\psi(t)$ itself.

Another procedure for solving for $K(t)$ is described in appendix B of reference [6]. This procedure is more accurate than the one described here in that it uses spline fits to $\psi(t)$ to estimate derivatives and it uses a more accurate integration scheme. However, when the procedure we use is stable, the results of the two methods agree closely.

I wish to thank Drs. Livingston and Dardy for making their data available to me prior to publication. 


\section{References}

[1] Zwanzig, R., in Lectures in Theoretical Physics, Eds. W. E. Brittin, W. B. Downs and J. Downs, (Interscience, New York, 1961), p. 106.

[2] Berne, B. J., Boon, J. P. and Rice, S. A., J. Chem. Phys. 45, 1086 (1966).

[3] van Konynenburg, P. and Steele, W. A., J. Chem. Phys. 56, 4776 (1972). This article contains numerous references to other work employing time correlation functions.

[4] Résibois, P., Brocas, J. and Decan, G., J. Math. Phys. 10, 964 (1969).

[5] Corngold, N., and Duderstadt, J. J., Phys. Rev. A 2, 836 (1970).

[6] Berne, B. J., and Harp, G. D., Adv. in Chem. Phys. XVII, $63(1970)$

[7] Harp, G. D., and Berne, B. J., Phys. Rev. A 2, 975 (1970).
[8] Rahman, A. in Statistical Mechanics, New Concepts, New Problems, New Applications, Eds. S. A. Rice, K. F. Freed and J. C. Light (U. of Chicago Press, Chicago, Ill., 1972), pp. 177-199.

[9] Kushick, J., and Berne, B. J., J. Chem. Phys. 59, 3732 (1973).

[10] Levesque, D. and Verlet, L., Phys. Rev. A 2, 2514 (1970).

[11] See for example, Table I of Ref. [7].

[12] Sköld, K., Rowe, J. M., and Ostrowski, G., and Randolph, P. D., Phys. Rev. A 6, 1107 (1972).

[13] Livingston, R. C., Rothschild, W. G., and Rush, J. J., J. Chem. Phys. 59, 2498 (1973).

[14] Dardy, H. D., Volterra, V., and Litovitz, T. A., J. Chem. Phys. 59, 4491 (1973).

[15] Wheelon, A. D., J. Math. and Phys. 34, 182 (1955).

[16] Ragman, A., J. Chem. Phys. 45, 2585 (1966).

(Paper 78A3-822) 